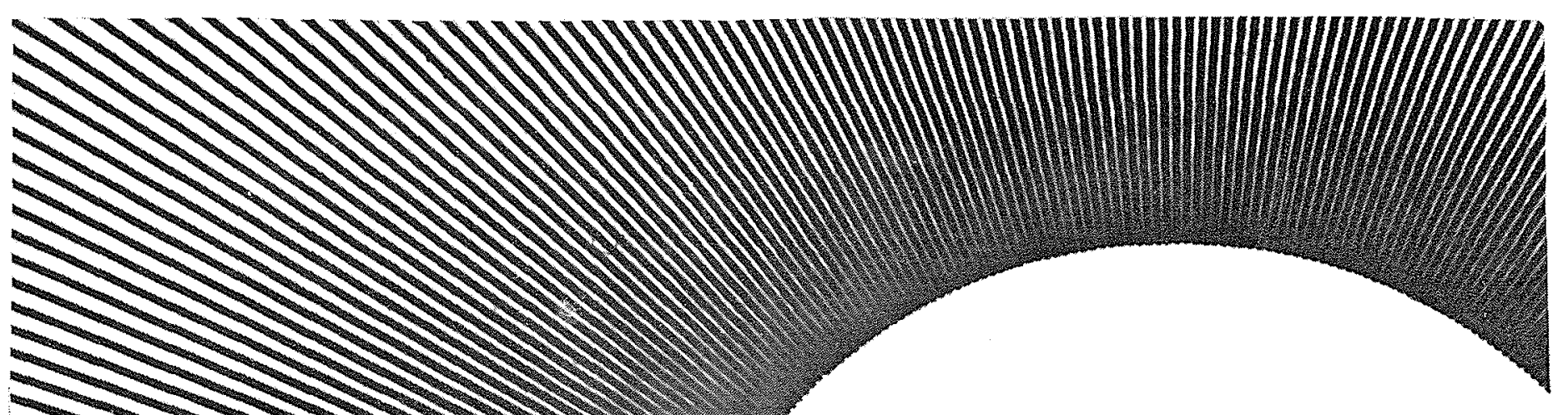

\title{
ALIMENTATION \\ EN EAU POTABLE DE L'ILE D'YEU
}

\author{
par G. BESNIER
}

Ingénieur Général du Génie Rural, des Eaux et des Forêts, Nantes

Le département de la Vendée comporte deux îles, Yeu et Noirmoutier, et la desserte des îles : transport des passagers et marchandises, alimentation en eau et en énergie pose des problèmes difficiles et coûteux.

Mon propos ne concernera que l'alimentation en eau de l'île d'Yeu mais je donnerai également quelques indications sur Noirmoutier car ce sont les techniques employées pour alimenter cette île qui nous ont guidés pour la desserte de l'île d'Yeu.

Noirmoutier se trouve séparée du continent en son extrémité sud par un bras de mer de $500 \mathrm{~m}$ de large aux marées basses de vive eau. Ce bras de mer s'élargit vers le nord et atteint $4,500 \mathrm{~km}$ au milieu de l'île mais il s'assèche à mimarée et comporte une chaussée carrossable «Le Gois», submersible à marée haute.

Les besoins en eau justifiaient la pose d'une canalisation de $200 \mathrm{~mm}$.

Initialement, nous avions pensé utiliser la chaussée du Gois mais, outre les $3,500 \mathrm{~km}$ de partie sous-marine, ce tracé nécessitait le raccordement au réseau continental au travers d'un marais peu habité. Au contraire, le raccordement par le sud, outre la brièveté đu trajet sous-marin, permettait l'alimentation à partir d'un réseau dense alimentant deux agglomérations: Fromentine et Notre-Dame-deMonts.

Enfin, les difficultés inhérentes à un travail qui n'aurait pu être exécuté que quelques heures par marée entrầnaient une charge financière nettement supérieure.

Le schéma de la pose fut le suivant:

Dans l'île la pointe sud est constituée par des dunes de sable.
Une aire de lancement partant de la plage fut aménagée au bull-dozer au travers des dunes sur $800 \mathrm{~m}$ de longueur et $12 \mathrm{~m}$ de largeur. La canalisation, tubes de $200 \mathrm{~mm}$ en acier laminé sans soudure de $8,17 \mathrm{~mm}$ d'épaisseur fut soudée électriquement sur l'aire de lancement, vérifiée à l'air comprimé. Le revêtement de soie de verre carboplast fut reconstitué à chaque soudure et éprouvé au balai électrique à $10000 \mathrm{~V}$. Puis la canalisation fut placée sur des diabolos constitués par trois roues avec pneumatique dont une dans un plan vertical et deux à $45^{\circ}$ de part et d'autre. Les diabolos étaient placés tous les $25 \mathrm{~m}$. La prépondérance fondrière étant de $8 \mathrm{~kg} / \mathrm{m}$ la conduite fut munie de flotteurs, de simples futs ayant une portée en lourd de $125 \mathrm{~kg}$ et placés tous les $30 \mathrm{~m}$. Les flotteurs étaient solidaires des tubes par deux feuillards. Chaque feuillard était épinglé de deux détonateurs reliés à deux circuits électriques indépendants.

Lorsque la préparation fut terminée, un câble fut fixé à l'extrémité de la conduite et tiré au travers du goulet, passé sur une poulie de renvoi frappée sur la plage côté continent et attachée à la barre d'un tracteur se déplaçant parallèlement au rivage.

Les opérations de lancement commencèrent deux heures avant la fin du jusant, avec coordination par radio. Un camion retenait la conduite sur l'île de façon à freiner la descente.

A la fin du jusant la conduite avait atteint le continent, mais présentait une fièche vers l'aval sous l'effet du courant. Avec le flot et sous l'effet combiné du courant et de l'action du tracteur la conduite se redressait. Lorsqu'elle fut rectiligne, la mise à feu des détonateurs libérait les 


\section{G. BESNIER}

flotteurs et la canalisation s'immergeait rapidement à l'endroit prévu. Elle était aussitôt remplie d'eau pour assurer sa prépondérance fondrière.

Une visite par homme grenouille confirme l'existence d'une fosse de 25 à $30 \mathrm{~m}$ de largeur que la canalisation franchissait en élévation.

La conduite a assuré l'alimentation de l'île de 1958 à 1971. Mais la construction du Pont de Noirmoutier et en particulier les batardeaux nécessaires à l'exécution des piles ont modifié le régime des courants dans le goulet.

Il en est résulté un affouillement de la conduite entraînant finalement la rupture de celle-ci. Mais entre temps une canalisation avait été placée sur le pont en sorte que l'alimentation de l'île ne fut pas interrompue.

Deux ans après Noirmoutier, en 1960, notre Service s'attaquait à l'Ile d'Yeu.

L'lle d'Yeu, c'est un anticlynal granitique faillé en son milieu, de $2300 \mathrm{ha}, 7 \mathrm{~km}$ sur 3 , situé à $18 \mathrm{~km}$ au large.

4739 habitants se consacrent presque uniquement à la pêche et à ses conséquences, la conserverie.

C'est le port d'armement le plus important en ce qui concerne la pêche du thon.

L'eau douce est rare dans l'île; le socle primitif étant imperméable et le relief peu accusé ne permettent pas la construction d'un barrage.

Seules solutions, le dessalement de l'eau de mer et la conduite sous marine. Nous avons choisi la conduite, moins onéreuse d'exploitation.

Les besoins de l'Ile, 200 à $300 \mathrm{~m}^{3}$ par jour en moyenne et $1200 \mathrm{~m}^{3}$ en pointe pouvaient être assurés par une conduite de $150 \mathrm{~mm}$ fonctionnant gravitairement en temps normal et avec surpression en période de pointe.

La technique de Noirmoutier nous servit de base d'étude pour la réalisation.

\section{Le terrain}

Les fonds entre l'île et le continent sont sablonneux et peu profonds. Ils descendent peu au dessous de la cote 10 des cartes marines et comportent des alternances de sables coquillés et de tables rocheuses en calcaires tendres avec des dénivellations excédant rarement $1 \mathrm{~m}$, le plus souvent de 50 à $70 \mathrm{~cm}$. Les barres rocheuses les plus importantes devaient être déroctées à la mine avant les travaux de lancement.

Les atterrages sont constitués par des plaques de sable fin devant des dunes, tant sur le continent que sur l'île.

\section{La canalisation}

Les tubes en acier laminé sans soudure de $150 \mathrm{~mm}$ ont une épaisseur de $4 \mathrm{~mm}$ et un poids de $19,05 \mathrm{~kg} / \mathrm{m}$.

Vides, ils flottent dans l'eau de mer et leur moment d'inertie n'est pas considérable compte tenu des efforts de flexion auxquels ils étaient susceptibles d'être soumis pendant la pose.

Nous avons donc choisi des tubes renforcés de $6,35 \mathrm{~mm}$ d'épaisseur pesant $31,300 \mathrm{~kg} / \mathrm{m}$ et ayant, viđes, une prépondérance fondrière de $5.8 \mathrm{~kg} / \mathrm{m}$. Les tubes éprouvés en usine à $50 \mathrm{~kg} / \mathrm{cm}^{2}$ étaient livrés avec un revêtement $\mathrm{C}$ renforcé $6 \%$ de soie de verre et carboplast. Ainsi était résolu le double problème de la résistance mécanique de la conduite et de la prépondérance fondrière qui devait assurer sa stabilité sur le fond, malgré les courants de marée qui peuvent atteindre 4 nœuds.

\section{La réalisation}

Le schéma de Noirmoutier ne pouvait être réalisé ici sans modification du fait des $18 \mathrm{~km}$ à tirer.

Compte tenu de la topographie des lieux, nous avons adopté le schéma suivant:

- assemblage des tuyaux par $600 \mathrm{~m}$ sur l'aire de lancement;

- mise en place des éléments avec raccordement sur le rivage d'un élément au suivant;

- traction de l'ensemble assurée par le treuil d'un bateau.

Nous avions pensé initialement amener les éléments par flottaison, effectuer la soudure sur une barge en mer avec les éléments déjà posés et immergés.

Il est apparu qu'il était impossible avec la houle d'assurer l'immobilisation des deux tronçons à souder.

C'est pourquoi nous avons adopté la solution précitée qui conduisait finalement à remorquer en mer un élément flottant de $18 \mathrm{~km}$.

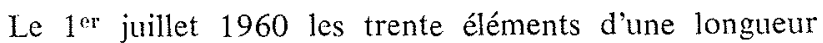
totale de $17553 \mathrm{~m}$ étaient alignés perpendiculairement à la plage sur une aire de lancement de $655 \mathrm{~m}$ sur $50 \mathrm{~m}$ de large ayant nécessité $26000 \mathrm{~m}^{3}$ de terrassement.

Chaque élément avait été éprouvé à l'air à $6 \mathrm{~kg} / \mathrm{cm}^{2}$ pendant $30 \mathrm{mn}$, puis à l'eau sous $35 \mathrm{~kg}$ pendant deux heures. Enfin une vérification avec balai électrique à $10000 \mathrm{~V}$ permettait de s'assurer de l'excellence du revêtement.

L'entreprise avait installé deux rampes de lancement identiques à celles de Noirmoutier et les éléments étaient répartis systématiquement de part et d'autre de la rampe de lancement.

Le side boon assurait la mise en place des éléments successivement sur l'un et l'autre chantier de lancement.

Le premier élément comportait une plaque pleine soudée avec un jeu de vannes permettant au choix de remplir la conduite d'eau ou d'air comprimé.

A leur mise en place sur les rampes les tubes étaient allégés par des flotteurs métalliques d'une portée en lourd de $156 \mathrm{~kg}$, placés tous les $30 \mathrm{~m}$ de façon à ce que la conduite vide dans l'eau conserve un poids apparent de $500 \mathrm{~g}$ au mètre.

Un premier essai fut tenté avec le navire l'Aiguillon, navire de $50 \mathrm{~m}$ équipé d'une machine à vapeur de $400 \mathrm{CV}$ et ancré à $5 \mathrm{~km}$ de la côte. Un câble reliait le treuil de l'Aiguillon à la conduite et des liaisons radios permettaient d'assurer une bonne coordination des opérations.

La première journée, $3 \mathrm{~km}$ de conduite furent ainsi tirées. Chaque élément une fois à l'eau était soudé sur la place de l'élément suivant.

Mais dès le lendemain, l'état de la mer interdisait tout travail et la conduite devait être immergée. L'été 1960 fut détestable et en octobre $4.700 \mathrm{~km}$, seulement avaient été lancés. L'opération fut arrêtée et reprise en avril 1961.

La conduite fut retirée entièrement pour vérification car les flotteurs n'avaient pu être largués. Les quelques blessures du revêtement au droit des flotteurs furent réparées et le premier juin l'opération était reprise.

L'Aiguillon avait été remplacé par le Cauville $55 \mathrm{~m}$, 225 tonneaux, 2 moteurs de $500 \mathrm{CV}$, trevil de $60 \mathrm{CV}$, avec un tambour de $900 \mathrm{~mm}$ de diamètre susceptible d'accueillir $8000 \mathrm{~m}$ de câble de $24 \mathrm{~mm}$ de diamètre et de développer un effort de 30 tonnes.

Le Cauville fut ancré à $7 \mathrm{~km}$ sur cinq coffres dont un dans l'axe.

Le câble fut déroulé mais allégé par des flotteurs de 120 li- 
tres de façon à éviter qu'il ne frotte sur le fond sur toute sa longueur.

L'opération fut donc reprise comme en 1960 et après traction du premier tronçon, le Cauville changea de mouillage et s'ancra ̀̀ 13 , puis à $17 \mathrm{~km}$ du continent.

A la fin de l'opération, le Cauville faisait demi tour et tirait sur le câble passant sur une poulie frappée sur le rivage de l'île et assurant ainsi l'arrivée de la conduite à destination.

Pendant toute l'opération les liaisons étaient assurées par trois postes radio de grande puissance et par un crisscraft muni de moteurs de $35 \mathrm{CV}$.

Commencée dès le premier juin 1961 à 15 h 45 l'opération était achevée le 14 juin à 20 h 15 .

Pendant toute la durée du lancement le contrôle des opérations était assuré par une équipe d'hommes grenouilles qui constataient que la conduite en équilibre indifférent se déplaçait de 30 à $40 \mathrm{~cm}$ du fond.

A la fin des opérations, les hommes grenouilles larguaient les flotteurs et plaçaient 60 ancres galvanisées de $60 \mathrm{~kg}$ réparties régulièrement le long de la conduite. Enfin, au droit des massifs rocheux les plus accidentés, des vieux pneus immobilisés par des sacs de ciment interdisaient tout déplacement de la conduite.

La conduite a été mise en eau aussitôt et reliée au réseau de l'île en cours de construction.

Avant de donner quelques détails sur la partie hydraulique, je voudrais achever ce qui concerne la conduite par la protection cathodique.

La protection cathodique est une vieille amie de notre service puisqu'en 1950 nous assurions la protection de la conduite de $72 \mathrm{~km}$ en acier de $500 \mathrm{~mm}$ qui alimente partiellement I.a Rochelle en eau à partir des barrages vendéens.

La protection cathodique est assurée par deux redresseurs dont les bornes négatives sont reliées à la conduite, les bornes positives étant reliées à une série de vieux rails enterrés à proximité.

Chaque poste situé aux deux extrémités de la conduite peut ainsi débiter 40 ampères sous douze volts avec un réglage possible du potentiel.

Le potentiel de la conduite a été mesuré par référence à l'électrode de Calomel qui donne les meilleurs résultats en milieu salé et en utilisant la méthode d'opposition.

Avec une tension aux bornes de $5,7 \mathrm{~V}$ et une intensité de 15 ampères, le potentiel de la conduite est abaissé de $2650 \mathrm{~m} / \mathrm{V}$.

Sur l'île, la tension appliquée est de $7,5 \mathrm{~V}$, le débit de 6,7 ampères et le potentiel abaissé de 2400 millivolts.

Le seuil de protection cathodique est largement atteint.

Les mesures ont lieu quatre fois par an. Elles ne comportent qu'un simple relevé de la tension aux bornes et d'intensité débitée mesurée par les appareils du tableau.

Depuis 1961, ces intensités sont constantes ce qui indique une bonne santé du revêtement de la conduite.

Statistiquement on pourrait dire que la résistance de la conduite est de $2800 \mathrm{ohms}$ par $\mathrm{m}^{2}$.

En réalité le potentiel de la conduite est variable tout le long de la conduite et on peut le calculer à partir de la résolution d'une équation différentielle.

En chaque point la chute de potentiel le long d'un élément $d x$ est:

$$
d \mathrm{E}=\mathrm{RI} d x
$$

$\mathrm{R}$ étant la résistance unitaire longitudinale de la conduite.
D'autre part, au point $x$ la variation d'intensité résulte de l'équation différentielle

$$
d \mathrm{I}=\mathrm{KE} d x
$$

$K$ étant la résistance d'isolement de la surface unitaire de la conduite en contact avec le milieu extérieur.

De la combinaison de ces deux équations différentielles on tire une équation différentielle du second ordre à coefficients constants :

$$
\frac{\mathrm{I}}{\mathrm{R}} \frac{d^{2} \mathrm{E}}{d x^{2}}-\mathrm{KE}=0
$$

qui admet pour solution la fonction chaînette.

On obtient les coefficients de cette fonction en se donnant le potentiel au point le plus éloigné de la source d'injection du courant.

Ce potentiel doit être au moins égal à -900 millivolts, d'où on déduit la valeur du potentiel à l'origine.

En ce qui concerne la conduite de l'Ile d'Yeu nous avions les éléments fournis par la conduite de Noirmoutier qui se trouvait dans des conditions analogues et c'est ainsi que nous avons pu déterminer le potentiel à appliquer au départ du continent.

Quand la conduite fut mise en service, nous nous sommes trouvés confrontés à une autre difficulté

Le débit de la conduite était variable dans de très grandes proportions et pour des raisons d'économic du projet, il n'était pas souhaitable de réaliser immédiatement le réservoir de mise en charge dans l'ile.

La pose d'un surpresseur devait assurer le débit aux heures de pointes, mais son fonctionnement risquait de provoquer dans le réseau de lîle des surpressions dangereuses. Il fallait donc que la marche du surpresseur soit fonction du débit demandé.

Le dispositif suivant fut adopté.

La conduite alimente normalement lîle, le débit étant mesuré par un compteur à impulsions.

Sur le trajet de la conduite se trouve placé un clapet anti-retour équilibré.

En aval du compteur une dérivation alimente une pompe d'un débit de $15 \mathrm{l} / \mathrm{s}$ sous une hauteur manométrique de $122 \mathrm{~m}$.

La pompe débite dans la conduite à l'aval du clapet antiretour.

D'autre part, l'entrée et la sortie de la pompe sont réunies par une conduite munie d'un clapet auto-stabilisateur type Lézier.

En temps normal, l'île est alimentée par gravité.

Dès que le compteur à tête émettrice enregistre un débit supérieur à $41 / \mathrm{s}$, les groupes électro-pompes se mettent en route et le clapet anti-retour se ferme automatiquement.

Le clapet régulateur de pression agit automatiquement de façon à ce que la pression manométrique à l'aval se tienne dans des limites compatibles avec la bonne tenue des canalisations.

Ce dispositif permet de faire varier le débit de 4 à $15 \mathrm{l} / \mathrm{s}$, la pression étant maintenue sensiblement constante à l'origine de la conduite.

Ce dispositif a fonctionné pendant plusieurs années à l'entière satisfaction des usagers jusqu'à la mise en service du réservoir d'accumulation situé dans l'île. 


\section{G. BESNIER}

Discussion

Président : M. D. LavaL

M. Ie Président remercie M. Bisnicr pour la clarté et la précision de son exposé; il félicite l'Administration du Génie Rural d'avoir si bien su s'adapter à un problème maritime ainsi que l'Entreprise Armor qui a réalisé avec maestria des opérations fort complexes.

M. GaLlioz demande si les matériaux modernes comme le polyester armé, permettent de réaliser des tuyaux aussi bien qu'en acier et donnant toute garantie contre la corrosion.

M. BesNrer estime que c'est avant tout une question de poids. Quant à la résistance de ces nouveaux matériaux, l'expérience a montré qu'au cours de leur mise en place les conduites n'ont pas été détériorées. Par contre, on manque d'observations de longue durée sur leur évolution dans le temps.

M. Richard confirme que le polyéthylène, le polychlorure de vinyle, les polyesters armés peuvent trouver leur emploi dans certaines conditions (effuents corrosifs, par exemple). Toutefois, en ce qui concerne les canalisations d'eau ordinaire, c'est jusqu'ici l'acier qui a donné les meilleurs résultats. Les canalisations en polychlorure de vinyle, posées en mer n'ont pas domé de grande satisfaction.

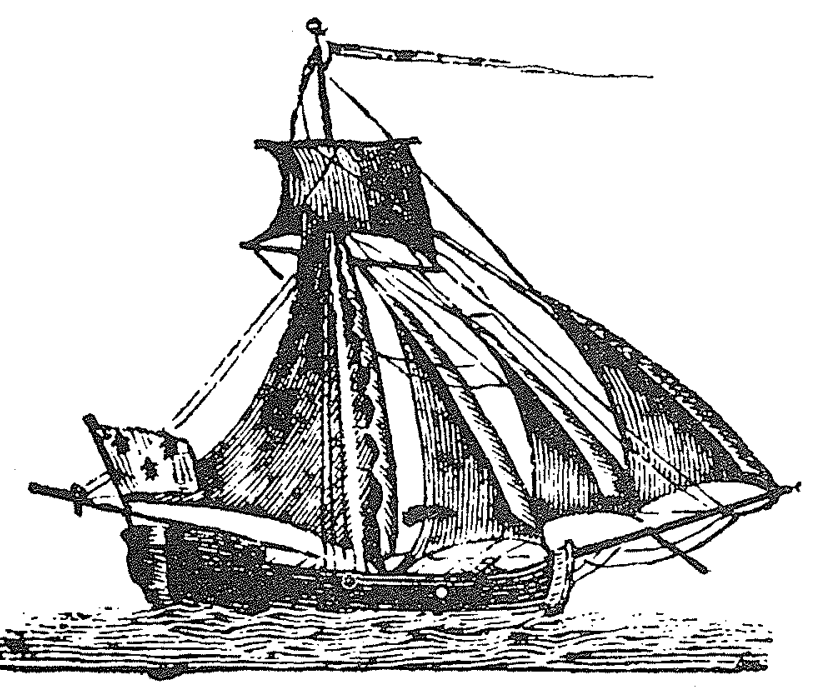

